\title{
Agenesia del Tercer Molar en Jóvenes entre 14 y 20 Años de Edad, Antofagasta, Chile
}

\author{
Third Molar Agenesis in Young People Between 14 and 20 Years of Age, Antofagasta, Chile \\ *Fernando García-Hernández; ** Oscar Toro Yagui; *"Manuel Vega Vidal \& ** Mauricio Verdejo Meneses
}

GARCÍA-HERNÁNDEZ, F.; TORO Y. O.; VEGA, V. M. \& VERDEJO M. M. Agenesia del tercer molar en jóvenes entre 14 y 20 años de edad, Antofagasta, Chile. Int. J. Morphol., 26(4):825-832, 2008.

RESUMEN: Se define agenesia como la ausencia de dientes por alteraciones genéticas aisladas o sindrómicas. La agenesia del tercer molar está asociada a malformaciones y considerada por diversos autores, consecuencia de la evolución humana. Son los dientes con mayor prevalencia de agenesia junto con segundos premolares e incisivos laterales. La prevalencia varía entre $9 \%$ y $37 \%$, en tanto, Arboleda et al. (2006) señalan una prevalencia del 20\%. La literatura señala variables estadísticas porcentuales, por género, por arcada dentaria, por lado y por diente, con escasos artículos sobre grupos originarios de Chile. La población en estudio consistió en 400 jóvenes (200 hombres y 200 mujeres) entre 14 y 20 años de edad pacientes de una clínica de ortodoncia de la ciudad de Antofagasta, Chile. Todos los pacientes debían ser sanos, sin ningún tipo de malformación general ni maxilofacial, que no hubiesen presentado enfermedades infecciosas que alteraran la odontogénesis y los períodos de erupción, sin exodoncias de ningún tercer molar y sin tratamiento ortodóncico previo al examen radiográfico panorámico. Se determina un $24,75 \%$ de casos de agenesia, con un porcentaje de agenesia respecto del número total terceros molares de un $12,63 \%$ y un $1,58 \%$ de agenesia de terceros molares, en relación al total de dientes. Predomina la agenesia en el género femenino, a nivel mandibular, en el lado derecho, de tipo doble y siendo el diente prevalente el tercer molar mandibular derecho, sin presentarse diferencias estadísticas significativas al 95\% de confianza.

PALABRAS CLAVE: Antropología; Odontología; Agenesia; Tercer molar.

\section{INTRODUCCIÓN}

La agenesia o anodoncia parcial es la ausencia congénita de uno o más dientes, asociada a síndromes, desórdenes genéticos únicos o aislada, sin componente genético.

Jorgenson (1980) la señala como un carácter poligénico hereditario, que es observable en miembros de una misma familia (Woelfel \& Scheid, 1998). Vastardis (2000) informa sobre la identificación de genes alterados responsables de la no formación de terceros molares. Kolenc (2004), Klein et al. (2005), Briceño et al. (2006) y TallónWalton et al. (2007) consideran que, como probable causa genética de agenesia familiar en molares, sería la mutación de los genes MSX1 y PAX9.

Es conocida la teoría de la reducción terminal dentaria de Adloff (apud Figún \& Garino, 1992 op cit) quien señala la futura desaparición del tercer molar en la especie humana, aspecto evolutivo consecuencia de la disminución de la actividad masticatoria. Es una línea evolutiva hacia un número menor de dientes (Shafer et al., 1977). En el 2001,
Proffit, citado por Arboleda et al., (2006), indica que las tendencias evolutivas han influido en la dentición actual y en la dimensión de los maxilares, en tanto que Bailit (1975) sugiere que la fórmula dentaria futura podría excluir a incisivos laterales, segundos premolares y terceros molares.

Según señalan Figún \& Garino, la evolución de los hábitos alimenticios ha contribuido a modificar el tamaño de los maxilares, siendo marcado este cambio a nivel mandibular, aspecto evolutivo del Homo sapiens sapiens que se confirma por la disminución progresiva del espacio retromolar entre borde anterior de la rama y cara distal del tercer molar, lo que actualmente se determina mediante la clasificación de Pell \& Gregory (que mide el espacio que tiene el tercer molar para erupcionar entre borde anterior de rama y cara distal del segundo molar) (Sandhu \& Kaur, 2005).

Sin embargo, autores como Rozkovcová et al. (1999) sugieren que "la agenesia no debe considerarse como una manifestación de la reducción filogenética del número de

\footnotetext{
* Unidad de Anatomía, Departamento Biomédico, Facultad de Ciencias de la Salud, Universidad de Antofagasta, Antofagasta, Chile.

** Cirujano Dentista, Clínica de Ortodoncia Manquehue, Antofagasta, Chile.
} 
dientes sino como una anomalía del desarrollo, producto de un proceso de mutación y selección basado en la herencia".

La causa de esta patología se debe a una alteración durante el proceso de formación de la lámina dentaria, que comienza entre el quinto mes de vida intrauterina y el nacimiento.

La agenesia compromete a varios dientes de la dentición humana y la literatura señala que son los terceros molares los que demuestran una mayor prevalencia (Fuller \& Denehy, 1984; Woelfel \& Scheid; Lozada, 2003; Hellman, citado por Bartolomé (1993); Sarmiento \& Herrera, 2004), siendo el orden habitual de los dientes ausentes el siguiente: terceros molares, premolares maxilares y mandibulares e incisivos laterales (Fuller \& Denehy).

Al respecto, la revisión de la literatura muestra una multiplicidad de trabajos referidos a la agenesia dentaria en general, muchos de los cuales excluyen a los terceros molares por su gran variabilidad.

Nanda (1954), señalaba que en la literatura no existe uniformidad en la recolección de datos y en la interpretación de los resultados, respecto de agenesias en terceros molares, situación que actualmente se mantiene y que podemos confirmar luego de revisar más de un centenar de publicaciones, lo que dificulta la comparación objetiva de los resultados, aunque es el diente con mayor prevalencia de agenesia.

Existe una gran variabilidad en los datos respecto de las diferencias por género, en el número de agenesias, en la arcada y lado predominante, en los porcentajes de agenesia, etc. McNamara \& Foley (2006) señalan que la hipodoncia del tercer molar tiene una prevalencia entre 9 y $37 \%$, mientras Arboleda et al., señalan una prevalencia de agenesia poblacional del $20 \%$, coincidiendo en que existe una mayor frecuencia en mujeres respecto de hombres, con una relación de $3: 2$.

Siendo el tercer molar el diente que provoca mayores patologías asociadas con su ausencia, retención, erupción y variabilidad de posición una vez erupcionado y cuyo síntoma principal es el dolor que produce, así como las dificultades quirúrgicas durante su exodoncia y las complicaciones postquirúrgicas (Cauvi \& Feldman, 1989; Ahlqwist
\& Gröndahl, 1991; Feldman et al., 1995; Martínez et al., 1995; Punwutikorn et al., 1999), efectuamos un estudio anátomo radiológico para determinar el grado de agenesia de terceros molares, según casos detectados y número total de terceros molares, su variabilidad según género, arcada y lado predominante en una muestra poblacional de jóvenes de edades entre 14 y 20 años de la ciudad de Antofagasta, con la finalidad de comparar resultados en grupos étnicos similares y para su aplicación clínica.

\section{MATERIAL Y MÉTODO}

Para la presente investigación se revisaron alrededor de 3.000 fichas clínicas desde el archivo de la Clínica de Ortodoncia Manquehue de Antofagasta, eligiendo 400 casos de individuos jóvenes, entre 14 y 20 años de edad, de ambos géneros (200 hombres y 200 mujeres). Las radiografías panorámicas debían corresponder a la primera radiografía diagnóstica de pacientes sanos, sin ningún tipo de malformación general ni maxilofacial, que no hubiesen presentado enfermedades infecciosas que alteraran la odontogénesis y los períodos de erupción, sin exodoncias de ningún tercer molar y sin tratamiento ortodóncico previo al examen radiográfico (Tabla I).

Se determinaron los 14 años como edad de inicio, ya que el tercer molar debe considerarse agenésico cuando está ausente después de esa edad, como expresan claramente Tavajoni-Kermani et al. (2002), lo cual es refrendado por Peck et al. (1996), quienes señalan que los 14 años corresponden a la "edad crítica" para observar la formación del tercer molar.

De los casos seleccionados se registraron los siguientes datos: nombre, edad, fechas de nacimiento y del examen radiográfico, historia clínica relevante, presencia de agenesias, grado de formación (clasificación de Nolla) y nivel de erupción (clasificaciones de Pell-Gregory \& de Winter) de los terceros molares, que fueron vertidos a un protocolo de investigación, tomando registro fotográfico de cada radiografía panorámica.

Los datos fueron tabulados en Microsoft Excel y para el análisis estadístico se empleó programa Statgraphics Plus 5.1

Tabla I. Características de la muestra.

\begin{tabular}{lcccc}
\hline \multicolumn{1}{c}{ Género } & N & Edad & Rango edad & $\%$ \\
\hline Masculino & 200 & 16 años 1 mes (193,65 meses) & 14 años-20 años 11 meses & 50,0 \\
Femenino & 200 & 16 años 1 mes (193,01 meses) & 14 años-20 años 11 meses & 50,0 \\
Total & 400 & 16 años 1 mes (193,33 meses) & 14 años-20 años 11 meses & 100,0 \\
\hline
\end{tabular}




\section{RESULTADOS}

Los resultados de agenesia determinada para la muestra de jóvenes de 14 a 20 años de edad, de la ciudad de
Antofagasta, Chile, se detalla en las Tablas II a VII).

Tabla II. Distribución porcentual de agenesia de terceros molares en una muestra de jóvenes de 14 a 20 años de edad de la ciudad de Antofagasta, Chile ( $\mathrm{n}=400)$.

\begin{tabular}{|c|c|c|c|c|c|c|c|c|}
\hline \multicolumn{3}{|c|}{$\begin{array}{c}\text { Agenesia según número casos en } \\
\text { estudio } n=400\end{array}$} & \multicolumn{3}{|c|}{$\begin{array}{c}\text { Agenesia según número total terceros } \\
\text { molares } n=1.600\end{array}$} & \multicolumn{3}{|c|}{$\begin{array}{l}\text { Agenesia de terceros molares según } \\
\text { número total de dientes } n=12.800\end{array}$} \\
\hline Según género & $\mathrm{N}^{\circ}$ casos & $\%$ & S egún género & $\mathrm{N}^{\mathrm{o}}$ casos & $\%$ & Según género & $\mathrm{N}^{\mathrm{o}}$ casos & $\%$ \\
\hline Masculino & $45 / 200$ & $22,5 *$ & Masculino & $91 / 800$ & $11,38 * *$ & Masculino & $91 / 6400$ & 1,42 \\
\hline Femenino & $54 / 200$ & 27,0 & Femenino & $111 / 800$ & 13,87 & Femenino & $111 / 6400$ & $1,73 * * *$ \\
\hline Total & $99 / 400$ & 24,75 & Totales & $202 / 1600$ & 12,63 & Totales & $202 / 12800$ & 1,58 \\
\hline
\end{tabular}

p-Valor $=0,297072$ sin diferencia estadísticamente significativa al 95\% al comparar porcentajes de casos de agenesia de terceros molares según género

** $\mathrm{p}$-Valor $=0,133767$ sin diferencia estadísticamente significativa al 95\% al comparar porcentajes del número de terceros molares agenésicos según género.

*** -Valor $=0,158995$ sin diferencia estadísticamente significativa al 95\% al comparar porcentajes de agenesia de terceros molares respecto del total de dientes según género

Tabla III. Agenesia de terceros molares según arcada y número total de terceros molares, en una muestra de jóvenes de 14 a 20 años de edad, de la ciudad de Antofagasta, Chile ( $\mathrm{n}=400)$.

\begin{tabular}{lcccc}
\multicolumn{2}{c}{ Género } & Maxilar & \multicolumn{2}{c}{ Mandíbula } \\
& $\mathbf{N}^{\mathbf{0}}$ casos & $\boldsymbol{\%}$ & $\mathbf{N}^{\mathbf{0}}$ casos & \% \\
\hline Masculino & $41 / 91$ & $45,1^{*}$ & $50 / 91$ & $54,9 * *$ \\
Femenino & $52 / 111$ & 46,8 & $59 / 111$ & 53,2 \\
Totales & $93 / 202$ & $46,0^{* * *}$ & $109 / 202$ & 54,0 \\
\hline
\end{tabular}

p-Valor $=0,809405$ sin diferencia estadísticamente significativa al 95\% al comparar arcada maxilar entre hombres-mujeres.

p-Valor $=0,809405$ sin diferencia estadísticamente significativa al 95\% al comparar arcada mandibular entre hombres-mujeres.

**** $\mathrm{p}$-Valor $=0,107839$ sin diferencia estadísticamente significativa al 95\% al comparar arcadas maxilar y mandibular en total de la muestra.

$\mathrm{p}$-Valor $=0,186137$ sin diferencia estadísticamente significativa al $95 \%$ al comparar ambas arcadas en hombres.

p-Valor $=0,340296$ sin diferencia estadísticamente significativa al 95\% al comparar ambas arcadas en mujeres.

Tabla IV. Distribución porcentual de agenesia según tercer molar ausente de la arcada, en una muestra jóvenes de 14 a 20 años de edad, de la ciudad de Antofagasta, Chile ( $\mathrm{n}=400)$.

Hombres

\begin{tabular}{cccccccc}
\hline & \multicolumn{3}{c}{ Maxilar } & \multicolumn{3}{c}{ Mandíbula } \\
\hline \multicolumn{2}{c}{ Diente 1.8} & \multicolumn{2}{c}{ Diente 2.8} & \multicolumn{2}{c}{ Diente 3.8} & \multicolumn{2}{c}{ Diente 4.8} \\
\hline $\mathrm{N}^{\circ}$ casos & $\%$ & $\mathrm{~N}^{\circ}$ casos & $\%$ & $\mathrm{~N}^{\circ}$ casos & $\%$ & $\mathrm{~N}^{\circ}$ casos & $\%$ \\
$23 / 91$ & 25,3 & $18 / 91$ & 19,8 & $21 / 91$ & 23,1 & $29 / 91$ & $31,9 *$
\end{tabular}

Mujeres

\begin{tabular}{|c|c|c|c|c|c|c|c|}
\hline \multicolumn{4}{|c|}{ Maxilar } & \multicolumn{4}{|c|}{ Mandíbula } \\
\hline \multicolumn{2}{|c|}{ Diente 1.8} & \multicolumn{2}{|c|}{ Diente 2.8} & \multicolumn{2}{|c|}{ Diente 3.8} & \multicolumn{2}{|c|}{ Diente 4.8} \\
\hline $\mathrm{N}^{\mathrm{o}}$ casos & $\%$ & $\mathrm{~N}^{\mathrm{o}}$ casos & $\%$ & $\mathrm{~N}^{\circ}$ casos & $\%$ & $\mathrm{~N}^{\mathrm{o}}$ casos & $\%$ \\
\hline $25 / 111$ & 22,6 & $27 / 111$ & 24,3 & $32 / 111$ & $28,8 * *$ & 27/111 & 24,3 \\
\hline \multicolumn{8}{|c|}{ Total hombres y mujeres } \\
\hline
\end{tabular}

\begin{tabular}{cccccccc}
\hline \multicolumn{3}{c}{ Maxilar } & \multicolumn{3}{c}{ Mandíbula } \\
\hline \multicolumn{2}{c}{ Diente 1.8} & \multicolumn{2}{c}{ Diente 2.8} & \multicolumn{2}{c}{ Diente 3.8} & \multicolumn{2}{c}{ Diente 4.8} \\
$\mathrm{~N}^{\mathrm{o}}$ casos & $\%$ & $\mathrm{~N}^{\mathrm{o}}$ casos & $\%$ & $\mathrm{~N}^{\mathrm{o}}$ casos & $\%$ & $\mathrm{~N}^{\circ}$ casos & $\%$ \\
$48 / 202$ & 23,8 & $45 / 202$ & 22,3 & $53 / 202$ & 26,2 & $56 / 202$ & $27,7 * * *$ \\
\hline
\end{tabular}

" $\mathrm{p}$-Valor $=0,0622856$ sin diferencia estadísticamente significativa al 95\% al comparar en hombres porcentaje de agenesia diente 4.8 con porcentaje de agenesia diente 2.8 * p-Valor $=0,117985$ sin diferencia estadísticamente significativa al 95\% al comparar en mujeres porcentaje de agenesia diente 3.8 con porcentaje de agenesia diente 1.8 .

$* *$ p-valor $=0,210097$ sin diferencia estadísticamente significativa al $95 \%$ al comparar en la muestra total porcentaje de agenesia diente 4.8 con porcentaje de agenesia diente 2.8 . 
GARCíA-HERNÁNDEZ, F.; TORO Y. O.; VEGA, V. M. \& VERDEJO M. M. Agenesia del tercer molar en jóvenes entre 14 y 20 años de edad, Antofagasta, Chile. Int. J. Morphol.,26(4):825-832, 2008.

Tabla V. Distribución porcentual de agenesia según lado y género terceros molares, en una muestra jóvenes de 14 a 20 años de edad, de la ciudad de Antofagasta, Chile ( $\mathrm{n}=400)$.

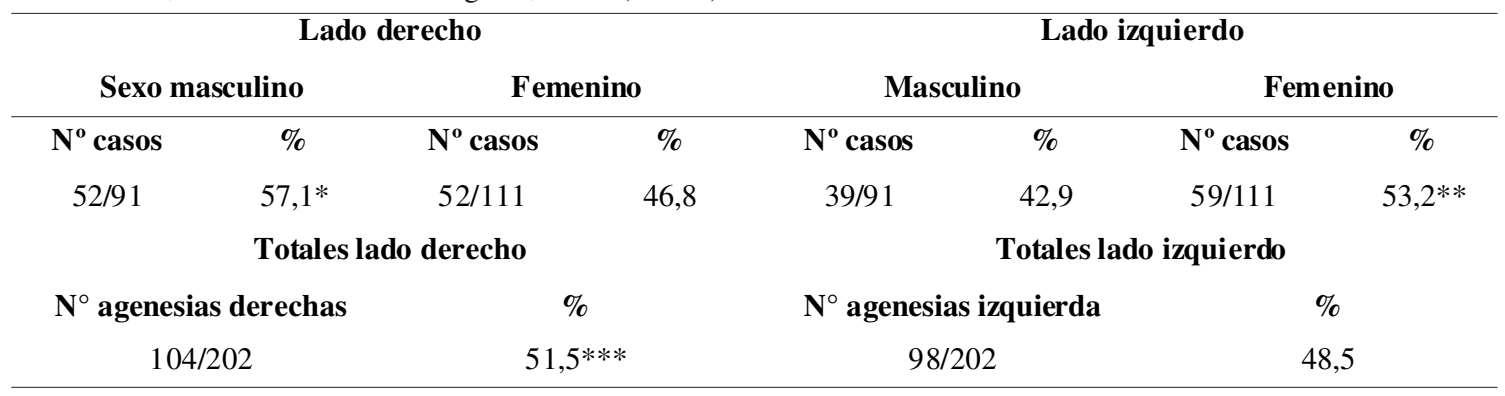

p-Valor $=0,0554049$ sin diferencia estadísticamente significativa al 95\% al comparar porcentaje de agenesia según lado en género masculino. ** $\mathrm{p}$-Valor $=0,340296$ sin diferencia estadísticamente significativa al 95\% al comparar porcentaje de agenesia según lado en género femenino.

${ }^{* * *}$ p-Valor $=0,546511$ sin diferencia estadísticamente significativa al 95\% al comparar porcentaje de agenesia según lado en el total de la muestra.

Tabla VI. Distribución porcentual de agenesia según número de dientes ausentes y género de uno o más terceros molares, en una muestra jóvenes de 14 a 20 años de edad, de la ciudad de Antofagasta, Chile $(\mathrm{n}=400)$.

\begin{tabular}{lrrrrrc}
\hline \multicolumn{1}{c}{ Género } & \multicolumn{2}{c}{ Masculino } & \multicolumn{2}{c}{ Femenino } & \multicolumn{2}{c}{ Total } \\
\multicolumn{1}{c}{ Agenesia } & $\mathbf{N}^{\mathbf{0}}$ & $\boldsymbol{\%}$ & $\mathbf{N}^{\mathbf{0}}$ & $\boldsymbol{\%}$ & $\mathbf{N}^{\mathbf{0}}$ & $\%$ \\
\hline 1 molar & 15 & 33,3 & 20 & 37,0 & 35 & 35,4 \\
2 molares & 19 & 42,2 & 20 & 37,0 & 39 & $39,4^{*}$ \\
3 molares & 6 & 13,3 & 5 & 9,3 & 11 & 11,1 \\
4 molares & 5 & 11,2 & 9 & 16,7 & 14 & $14,1^{* *}$ \\
Total & 45 & 100 & 54 & 100 & 99 & 100 \\
\hline
\end{tabular}

"p-Valor $=0,0000045875$ con diferencia estadísticamente significativa al 95\% al comparar porcentaje de casos de agenesia de dos molares respecto porcentaje de casos de agenesia de tres terceros molares.

* $\mathrm{p}$-Valor $=0,0000579263$ con diferencia estadísticamente significativa al 95\% al comparar porcentaje de casos de agenesia de dos molares respecto porcentaje de casos de agenesia de cuatro terceros molares.

Tabla VII. Distribución de agenesia por género según número de terceros molares ausentes, en una muestra jóvenes de 14 a 20 años de edad, de la ciudad de Antofagasta, Chile (n=400).

\begin{tabular}{ccc}
\hline Masculino & Femenino & Total \\
\hline $2-1-3-4$ & $1-2-4-3$ & $2-1-4-3$ \\
\hline
\end{tabular}

\section{DISCUSIÓN}

Del análisis de los resultados obtenidos es posible deducir lo siguiente respecto de:

1) Porcentaje de individuos que presentan uno o más terceros molares con agenesia: El porcentaje de $24,75 \%$ de individuos con agenesia de terceros molares obtenido en nuestro trabajo, se encuentra dentro del rango de $20 \%-30 \%$ determinado por autores como Keene $(1965)=$ 27\%; Ortega Piga $(1987)=29 \%$; Shad \& Boyd $(1979)=$ 23,3\%; Thorson \& Häag $(1991)=23 \%$ y Bredy et al., (1991) $=20,7$ (todos citados por Bartolomé); Thompson et al. (1974) $=22,3 \% ;$ Hattab $(1995)=27,0 \% ;$ Lynham $(1990)=22,7 \%$;
Mok \& Ho $(1996)=28,5 \%$; Rozcovcová et al. $=22,5 \%$; Kruger et al. $(2001)=27,9 \%$; Baba-Kawano et al $.(2002)=$ 20,8\%; Sandhu \& Kaur $=24,0 \%$ y a nivel latinoamericano con los obtenidos en trabajos realizados por Cuairán et al. $(1996)=26,75 \%$ y Silva Meza $(2003)=24,3 \%$ en mexicanos; Sarmiento \& Herrera $=21,3 \%$ en colombianos; Castilho et al. $(1990)=20,39 \%$; Paula \& Ferrer $(2007)=20,18$ en brasileños.

García-Hernández \& Beltrán (2008) determinaron en una investigación realizada en una muestra de atacameños o Likan antai, grupo étnico originario del Norte de Chile un 
porcentaje de agenesia de $26,7 \%$, valor muy aproximado al obtenido en la presente investigación y con $\mathrm{p}=0,699904$, lo que indica que no existe diferencia estadísticamente significativa al $95 \%$.

Porcentajes menores a $20 \%$ fueron obtenidos por Gran et al., $(1963)=16,4 \%$ apud Bartolomé op cit; y a nivel de Latinoamérica Crispin et al. $(1972)=16,0 \%$, Nicodemo $(1973)=18,5 \%$, Oliveira \& Serra Negra $(1976)=14,42 \%$ (todos citados por Bartolomé); además de Oliveira \& Serra Negra $(1984,1985)=10,83 \%$ en brasileños, Henríquez $(1972)=15,0 \%$ (apud Bartolomé op cit); y Méndez \& Contreras $(2006)=15,36 \%$ en venezolanos; Altunaga et al . $(2008)=12,45 \%$ en cubanos; Bastidas \& Rodríguez (2004) $=10,6 \%$ en colombianos.

Mientras que porcentajes por sobre el 30\% fueron determinados por Goren et al (2005) $=38,5 \%$; Bartolomé $=$ 30,8; Lima de Castro et al. (2006) $=40,13 \%$ en brasileños y Feldman et al. $=44,75 \%$ en Chile.

En la Tabla VIII se muestran los autores que determinaron porcentajes en que existe diferencia estadísticamente significativa al $95 \%$ respecto del porcentaje obtenido en nuestra investigación.

2) Porcentaje de agenesia según número total de terceros molares: La literatura muestra rangos entre los 3,75\% y $29,89 \%$ de agenesia respecto del total de terceros molares, obteniendo en nuestro trabajo un valor intermedio de $12,63 \%$ de agenesia.

3) Porcentaje de agenesia según el número total de dientes: En relación al porcentaje de agenesia de terceros molares respecto del número total de dientes que debieran estar pre- sentes en la dentición humana adulta, la literatura señala rangos entre $1,70 \%$ y $19,4 \%$, estando nuestros resultados muy cercanos al rango menor al determinarse un porcentaje de $1,58 \%$ de agenesia.

4) Distribución por género: Predomina la agenesia en el género femenino $(27,0 \%-22,5 \%)$, sin diferencias estadísticas significativa al 95\%, coincidiendo con lo citado en la literatura en que las diversas investigaciones muestran algún predominio de uno u otro género sin diferencias estadísticas significativas al $95 \%$.

5) Arcada predominante: La agenesia mandibular predomina en todo el grupo en estudio $(54,0-46,0 \%)$ y lo mismo sucede según género, tanto en hombres $(54,9$ - 45,1\%) como en mujeres (53,8 - 46,2\%), sin diferencias estadísticas significativa al $95 \%$ de confiabilidad, según género y arcada. Los valores coinciden con lo citado en la literatura.

6) Lado predominante: En el análisis de la muestra total predominan los casos de agenesia derecha (51,5 - 48,5\%), de acuerdo a lo citado en la literatura que señala un mayor número de casos de agenesia derecha en relación a los terceros molares. Lo mismo se comprueba en el género masculino $(57,1-42,9 \%)$, mientras que las mujeres muestran un predominio de las agenesias izquierdas $(46,8-53,2 \%)$. Al realizar la comparación estadística de los diversos grupos en ninguno existe diferencia significativa al $95 \%$.

7) Molar agenésico predominante: En la muestra en estudio el tercer molar mandibular derecho (diente 4.8.) es el diente que muestra el mayor porcentaje de agenesia con un $27,7 \%$ y lo mismo se observa en el género masculino $31,9 \%$, en tanto en mujeres el diente con mayor porcentaje de agenesia es el tercer molar mandibular izquierdo (diente 3.8.)

Tabla VIII. Comparación de porcentajes de agenesia del tercer molar determinados por diversos investigadores que muestran diferencias estadísticamente significativas al 95\% respecto, de los valores obtenidos en nuestra investigación $(24,75 \%-n=400)$.

\begin{tabular}{llcccc}
\hline A utor & País & año & n & \% & $\begin{array}{c}\text { Diferencia estadística } \\
\text { significativa al 95\% p-valor= }\end{array}$ \\
\hline Crispim et al. & Brasil & 1972 & 490 & 16,0 & 0,00115271 \\
Henríquez & Venezuela & 1972 & 200 & 15,0 & 0,00613584 \\
Oliveira \& Serra Negra & Brasil & 1976 & 416 & 14,42 & 0,000195853 \\
Oliveir a \& Serra Negra & Brasil & 1984 & 120 & 10,83 & 0,00114073 \\
Altunaga et al. & Cuba & 2003 & 265 & 12,45 & 0,000098893 \\
Bastidas \& Rodríguez & Colombia & 2004 & 141 & 10,6 & 0,000395286 \\
Méndez \& Contreras & Venezuela & 2006 & 397 & 15,36 & 0,000936038 \\
Feldman et al. & Chile & 1995 & 143 & 44,75 & 0,00000751806 \\
Goren et al. & Israel & 2005 & 226 & 38,5 & 0,000299684 \\
Lima de Castro et al. & Brasil & 2006 & 224 & 40,13 & 0,0000603839 \\
\hline
\end{tabular}


con un porcentaje del $28,8 \%$. En todos los análisis no se comprobó diferencia estadística significativa al $95 \%$.

8) Número de agenesias por individuo: En el presente estudio se detectó un mayor número de casos con doble agenesia con un $39,4 \%$, mientras que el porcentaje de agenesias simples fue de $35,4 \%$, coincidiendo ambos valores con lo citado en la literatura, que indica el predominio de las agenesias simples o dobles. Se detectaron similar porcentaje de casos de triple y cuádruple agenesias $(11,1-14,1 \%)$.

\section{CONCLUSIONES}

1) Para el diagnóstico y los estudios evolutivos sobre la presencia o ausencia del tercer molar, los exámenes radiográficos deben realizarse a individuos mayores de 14 años de edad.

2) La existencia de variables porcentuales marcadas se comprueba con diversos trabajos referidos a la agenesia del tercer molar, algunos con valores muy próximos a los obtenidos en nuestro estudio y otros con variaciones porcentuales menores o mayores, siendo algunas de estas diferencias porcentuales estadísticamente significativas al 95\% (ver Tabla VIII).
3) La muestra 400 jóvenes de 14 a 20 años de edad, de la ciudad de Antofagasta (200 hombres y 200 mujeres), presenta agenesia de los terceros molares en un $24,75 \%$ de los casos, con un porcentaje de agenesia respecto del número total terceros molares de un $12,63 \%$ y de un $1,58 \%$ de agenesia de terceros molares en relación al total de dientes.

4) Predomina la agenesia en el género femenino, a nivel mandibular, en el lado derecho, de tipo doble y siendo el diente prevalente el tercer molar mandibular derecho (diente 4.8.), sin presentarse diferencias estadísticas significativas al $95 \%$ de confianza.

5) La presente investigación es un aporte al estudio antropológico y del sistema estomatognático de la población del Norte de Chile, permitiendo realizar comparaciones estadísticas con poblaciones de diferentes grupos étnicos y poblacionales.

\section{AGRADECIMIENTOS}

Los autores expresan sus agradecimientos a la administración y al personal de la Clínica de Ortodoncia Manquehue por su colaboración y ayuda para la realización del presente trabajo de investigación.

GARCÍA-HERNÁNDEZ, F.; TORO Y. O.; VEGA, V. M. \& VERDEJO M. M. Third molar agenesis in young people between 14 and 20 years of age, Antofagasta, Chile. Int. J. Morphol., 26(4):825-832, 2008

SUMMARY: Agenesis is defined as the absence of teeth by single or sindromics genetic alterations. Third molar agenesis is associated to malformations and is considered a consequence of human evolution by several authors. Third molar are the teeth with greater percentage of agenesis along with seconds premolars and lateral incisors. The frequency varies between $9 \%$ and $37 \%$, while Arboleda et al., (2006) indicate a $20 \%$ of agenesis. Literature indicates statistical variables percentage, by gender, dental arches, side and tooth, however few articles in original groups of Chile. The population in study consisted of 400 young people ( 200 men and 200 women) between 14 and 20 years of age patient of a clinic of orthodontia of the city of Antofagasta, Chile. All the patients had to be healthy, without any type of general and maxillofacial malformation, who had not presented infectious diseases that altered the odontogenesis and the periods of eruption, without exodontias of third molar and without previous orthodontic treatment to the panoramic x-ray examination. A $24.75 \%$ of cases of agenesis were determined, with a percentage of $12.63 \%$ of agenesis with respect to the total number of third molars and $1.58 \%$ of agenesis of third molar in relation to the total of teeth. Agenesis in the female predominates, at mandibular level, in the right side, of double type and being the prevalent tooth is mandibular right third molar, without appearing significant statistical differences to $95 \%$ of confidence.

KEY WORDS: Anthropology; Dentistry; Agenesis; Third molar.

\section{REFERENCIAS BIBLIOGRÁFICAS}

Ahlqwist, M. \& Gröndahl, H. G. Prevalence of impacted teeth and associated pathology in middle-age and older Swedish women. Community Dent. Oral Epidemiol. 19:116-9, 1991.

Altunaga, A.; Hidalgo, A.; Burgos, G. \& Puig, L. La oligodoncia un problema a tener en cuenta. http://
Www.monografías.com/trabajos 28/oligodoncia/ oligodoncia.shtml, 2008).

Arboleda, L. A.; Echeverri, J.; Restrepo, L. A.; Marín, M. L.; Vásquez, G.; Gómez, J. C.; Manco, H. A.; Pérez, C. M \& Taborda, E. Agenesia dental. Revisión bibliográfica y reporte de dos casos clínicos. Rev. Fac. Odontol. 
Univ. Antioq. 18(1):47-54, 2006.

Baba-Kowano, S.; Toyoshima, Y.; Regalado, L.; Sa'do, B. \& Nakasima, A. Relationship between missing coger third molar and late formation of tooth germs. Angle Orthod. 72:112-7, 2002.

Bailit, H. L. Dental variation among population. An anthropologic view. Dent. Clin. North Am. 19(1):12539, 1975

Bartolomé, B. Desarrollo y agenesia del tercer molar en una población de niños y adolescentes españoles. Tesis Doctoral. Universidad Complutense de Madrid, 1993.http:/ /www.cibernetia.com/tesis_es/Ciencias_Médicas/Cirugía/Estomatología_y_Ortodoncia/22, 2007).

Bastidas, M. A. \& Rodríguez, A. M. Agenesia dental en pacientes jóvenes. Revista Estomatología 12(2):34-43, 2004.

http://odontologia.univalle.edu.co/revista/publicaciones/1202-2004/pdf/04v12no2-04.pdf, 2007)

Briceño, I.; Berrocal, M. C.; González, O. A.; Gutiérrez, S. J.; Ordóñez, A. \& Torres, D. Mecanismo de herencia de la agenesia dental. http://www.encolombia.com/odontología/investigaciones/memorias-Vencuentroanalisis.htm, 2006.

Castilho, J. C. M.; Nicodemo, R. A.; Bazzarella, C. B \& Moraes, L. C de. Prevâlencia de anodontia entre estudiantes do $2^{\circ}$ Grau da cidade de Säo José dos Campos: correlacäo dessa anomalia entre terceiros molares e outros órgäo. Rev. Odontol. UNESP 19(1):269-76, 1990.

Cauvi, D. \& Feldman, I. Presencia clínica de los terceros molares y su relación con la pérdida de los primeros y/ o segundos molares permanentes. Rev. Iberoamericana de Ort. 9(1):9-25, 1989.

Cuairán, V.; Gaitán, L. A. \& Hernández, A. J. Agenesia dental en una muestra de pacientes ortodónticos del Hospital Infantil de México. Rev. ADM 53(4):211-5, 1996.

Feldman, I.; Estupiñán, C.; Cauvi, D.; Espinoza, A. \& Rajecic, Z. Estudio comparado de agenesia de terceros molares en pacientes figurados y no figurados. Rev. Fac. Odont. Univ. de Chile 13(1):26-35, 1995.

Figún, M. E. \& Garino, R. R. Anatomía odontológica funcional y aplicada. $2^{\text {a }}$ ed. Buenos Aires, El Ateneo, 1992. pp.341-2.
Fuller J. L. \& Denehy, G. E. Concise dental anatomy and morphology. Chicago, Year Book Publishers Inc., 1984. pp.264-5.

García-Hernández, F. \&, Beltrán, V. V. J. Agenesia del tercer molar en una etnia originaria del Norte de Chile: Atacameños o Lican Antai. Int. J. Morphol., 26(3):58390, 2008.

Goren, S; Tzoisner, R.; Dinbar, A.; Levin, L. \& Brezniak, N. Prevalence of congenitally missing teeth in Israeli recruits. Refuat Hapeh Vehashinaim 22(2):49-53, 2005.

Hattab, F. N. Impaction status of third molars in Jordanian students. Oral Surg. Oral Med. Oral Pathol. Oral Radiol. Endont. 79(1):24-9, 1995.

Jorgenson, R. J. Clinician's view of hypodoncia. J. Am. Dent. Assoc., 101:283-6, 1980.

Klein, M. L.; Nieminen, P.; Lammi, L.; Niebuhr, E. \& Kreiborg, S. Novel mutation of the initiation codon of PAX9 causes oligodontia. J. Dent. Res., 84(1):43-7, 2005.

Kolenc, F. J. Agenesias dentarias: en busca de alteraciones genéticas responsables de la falta de desarrollo. Med. Oral Patol. Oral Cir. Bucal 9(5):385-95, 2004.

Kruger, E.; Thompson, W. M. \& Konthasinghe, P. Third molar outcomes from age 18 to 26: Findings from population-based New Zealand longitudinal study. Oral Surg. Oral Med. Oral Pathol. Oral Radiol. Endod 92(2):150-5, 2001.

Lima de Castro, E. V. F.; Lima de Castro, A.; Pescinini, L. M.; Coelho, P. de T: \& Biazon, A. T. Agenesia e inclusao dental patológica. Estudo clínico e radiográfico em pacientes. Rev. Fac. Odontol. Lins, Piracicaba 18(1):416, 2006.

Lozada, O. Alteraciones en el número de dientes asociados a maloclusiones. Postgrado de Odontología, Facultad de Odontología, Universidad Central de Venezuela, Caracas, 2003.

Lynham, A. Ranoramic radiographic survey of hipodoncia in Australian Defence Force recruits. Aust. Dent. J. 35(1):19-22, 1990.

Martínez, S.; Concha, G. \& San Pedro, J. Estudio radiográfico de terceros molares inferiores en una muestra de individuos de 18 a 20 años. Rev. Fac. Odont. Univ. de Chile 13(1):43-9, 1995. 
McNamara, C \& Foley T. Multidisciplinary management of hypodontia in adolescents: case report. J. Can. Dent. Assoc. 72(8):740-6, 2006.

Méndez, D. C. \& Contreras, Ch. C. Anomalías de múmero en pacientes con dentición mixta. http:// w w w.ortodoncia.ws/publicaciones/2006/ anomalias_numero_pacientes_denticion_mixta.asp\#, 2006.

Mok, Y. Y. \& Ho, K. K. Congenitally absent third molars in 12 to 16 year old Singaporean Chinese patients: a retrospective radiographic study. Ann. Acad. Med. Singapore 25(6):828-30, 1996.

Nanda, R. S. Agenesis of the third molar in man. Am. J. Orthod., 40:698-706, 1954.

Oliveira, O. L. \& Serra Negra, E. Agenesia de terceiros molares em negros brasileiros. Arq. Centro Estad. Curso Odontol., 21/22(2/1):103-11, 1984-1985.

Paula, A. F. B. \& Ferrer, K. J. N. Prevalência de agenesia em uma clínica ortodôntica de Goiânia. RGO, Porto Alegre, 55(2):149-53, 2007.

Peck, S.; Peck, L. \& Kataja, M. Site-specificity of tooth agenesis in subjects with maxillary canine malpositions. Angle Orthod., 66(6):473-6, 1996.

Punwutikorn, J.; Waikakul, A. \& Ochareon, P. Synptoms of unerupted mandibular third molar. Oral Surg. Oral Med. Oral Pathol. Oral Radiol. Endod., 87(3):305-10, 1999.

Rozkovcová, E.; Markova, M. \& Dolefsi, J. Studies on agenesis of third molar among populations of different origins. Sb. Lek., 100(2):71-84, 1999.

Sandhu, S. \& Kaur, T. Radiographic evaluation of the status of the third molar in the Asian-Indian students. J. Oral Maxilofacial Surg., 63(5):640-5, 2005.

Sarmiento, P. \& Herrera, A. Agenesia de terceros molares en estudiantes de Odontología de la Universidad del Valle entre 16 y 25 años. Revista Colombia Médica, 35:(3):5$9,2004$.

Shafer, W.; Hine, M. \& Levy, B. A textbook of Oral Pathology. $4^{\text {th }}$ ed. Philadelphia, Saunders Company, 1977.

Silva Meza, R. Radiographic assessment of congenitally missing teeth in orthodontic patients. Int. J. Paediatr. Dent., 13(2):112-6, 2003.
Tallón-Walton, V.; Manzanares-Céspedes, M. C.; Sirpa, A.; Carvalho-Lobato, P; Valdivia-Gandur, I.; GarcíaSusperregui, A.; Ventura, F. \& Nieminen, P. Identification of a novel mutation in the PAX9 gene in a family affected by oligodontia and other dental anomalies. Eur. J. Oral Sci., 115:427-32, 2007.

Tavajoni-Kermani, H.; Kapur, R. \& Sciote, J. J. Tooth agenesis and craneofacial morphology in an orthodontic population. Am. J. Orthod Dentofacial Orthop., 122(1):39-47, 2002.

Thompson, G. W.; Popovich, F. \& Anderson, D. L. Third molar agenesis in the Burlington Growth Centre in Toronto. Community Dent. Oral Epidemiol., 2(4):18792, 1974.

Vastardis, H. The genetics of human tooth agenesis: new discoveries for understanding dental anomalies. Am. J. Orthod. Dentofacial Orthop., 117:650-6, 2000.

Woelfel, J. B. \& Scheid, R. C. Anatomía Dental. Aplicaciones Clínicas. Barcelona, Masson-Williams \& Wilkins, 1998.

Dirección para correspondencia:

Prof. Dr. Fernando García-Hernández

Unidad de Anatomía, Departamento Biomédico

Facultad de Ciencias de la Salud

Universidad de Antofagasta

Coquimbo 945, Depto 64

Antofagasta

CHILE

Recibido : 21-09-2008

Aceptado: 25-10-2008 